\title{
Age and gender interactions in short distance triathlon performance
}

Etter, Franziska ; Knechtle, Beat ; Bukowski, Arkadiusz ; Rüst, Christoph Alexander ; Rosemann, Thomas ; Lepers, Romuald

\begin{abstract}
This study investigated the participation and performance trends as well as the age and gender interaction at the Olympic distance 'Zürich Triathlon' (1.5 km swim, $40 \mathrm{~km}$ cycle and $10 \mathrm{~km}$ run) from 2000 to 2010 in 7,939 total finishers (1,666 females and 6,273 males). Female triathletes aged from 40 to 54 years significantly $(\mathrm{P}<$ 0.05 ) increased their participation while the participation of younger females and males remained stable. Males of 50-54 years of age and females of 45-49 years of age improved their total race time. For elite top five overall triathletes, mean gender differences in swimming, cycling, running and overall race time were $15.2 \pm 4.6 \%, 13.4$ $\pm 2.3 \%, 17.1 \pm 2.5 \%$, and $14.8 \pm 1.8 \%$, respectively. For both elite and age group athletes, the gender difference in cycling time was significantly $(\mathrm{P}<0.001)$ lower than for swimming and running. The gender difference in overall Olympic distance triathlon performance increased after the age of 35 years, which appeared earlier compared to long distance triathlon as suggested by previous studies. Future investigations should compare gender difference in performance for different endurance events across age to confirm a possible effect of exercise duration on gender difference with advancing age.
\end{abstract}

DOI: https://doi.org/10.1080/02640414.2012.760747

Posted at the Zurich Open Repository and Archive, University of Zurich

ZORA URL: https://doi.org/10.5167/uzh-73695

Journal Article

Accepted Version

Originally published at:

Etter, Franziska; Knechtle, Beat; Bukowski, Arkadiusz; Rüst, Christoph Alexander; Rosemann, Thomas; Lepers, Romuald (2013). Age and gender interactions in short distance triathlon performance. Journal of sports sciences, 31(9):996-1006.

DOI: https://doi.org/10.1080/02640414.2012.760747 


\section{Age and gender interactions in short distance triathlon performance}

\section{Short title: Age and gender interaction in triathlon}

Franziska Etter ${ }^{1}$, Beat Knechtle ${ }^{1,2}$, Arkadiusz Bukowski ${ }^{2}$, Christoph Alexander Rüst ${ }^{1}$, Thomas Rosemann ${ }^{1}$, Romuald Lepers ${ }^{3}$

1

Institute of General Practice and for Health Services Research, University of Zurich, Zurich, Switzerland

2 Gesundheitszentrum St. Gallen, St. Gallen, Switzerland

3 INSERM U1093, Faculty of Sport Sciences, University of Burgundy, Dijon, France

\section{Corresponding author}

PD Dr. med. Beat Knechtle

Facharzt FMH für Allgemeinmedizin

Gesundheitszentrum St. Gallen

Vadianstrasse 26

9001 St. Gallen

Switzerland

Telefon

+41(0) 712268282

Telefax

+41(0) 712268272

E-Mail:

beat.knechtle@hispeed.ch 


\begin{abstract}
This study investigated the participation and performance trends as well as the age and gender interaction at the Olympic distance 'Zürich Triathlon' $(1.5 \mathrm{~km}$ swim, $40 \mathrm{~km}$ cycle and $10 \mathrm{~km}$ run) from 2000 to 2010 in 7,939 total finishers (1,666 females and 6,273 males). Female triathletes aged from 40 to 54 years significantly $(P<0.05)$ increased their participation while the participation of younger females and males remained stable. Males of 50-54 years of age and females of 45-49 years of age improved their total race time. For elite top five overall triathletes, mean gender differences in swimming, cycling, running and overall race time were $15.2 \pm 4.6 \%, 13.4 \pm 2.3 \%, 17.1 \pm 2.5 \%$, and $14.8 \pm 1.8 \%$, respectively. For both elite and age group athletes, the gender difference in cycling time was significantly $(P<0.001)$ lower than for swimming and running. The gender difference in overall Olympic distance triathlon performance increased after the age of 35 years, which appeared earlier compared to long distance triathlon as suggested by previous studies. Future investigations should compare gender difference in performance for different endurance events across the age to confirm a possible effect of exercise duration on gender difference with advancing age.
\end{abstract}

Key words: gender difference - swimming - cycling - running - endurance 


\section{Introduction}

The gender difference in endurance sport performance has received considerable attention. Previous studies investigating the participation and performance trends of female endurance runners reported an increase in female participation and an improvement of their performance during the last three decades (Hoffman, 2010; Hoffman, \& Wegelin, 2009; Jokl, Sethi, \& Cooper, 2004; Lepers, \& Cattagni, 2012; Leyk, et al., 2007; Leyk, et al., 2009). Although some authors have questioned whether the gap in gender difference in endurance performance could be closed (Bam, Noakes, Juritz, \& Dennis, 1997; Coast, Blevins, \& Wilson, 2004; Whipp, \& Ward 1992), more recent studies could not confirm this assumption showing that the gender difference in both endurance performance (Cheuvront, Carter, DeRuisseau, \& Moffart, 2005; Sparling, O’Donnell, \& Snow, 1998) and anaerobic sprints (Seiler, De Koning, \& Foster, 2007) is no longer diminishing. Coast et al. (2004) for example analysed the gender difference in running performances at distances from $100 \mathrm{~m}$ to $200 \mathrm{~km}$. They found a gender difference in average speed of $12.4 \%$ and also showed that longer running distances were associated with greater gender differences. In contrast, Tanaka and Seals (1997) reported the percent gender difference in swimming performance became smaller with increasing distance, from $19 \%$ in $50 \mathrm{~m}$ to $11 \%$ in $1500 \mathrm{~m}$, respectively.

In contrast to endurance running, the gender difference in triathlon regarding participation and performance has been less investigated. However, triathlon involving successively swimming, cycling and running, represents an intriguing alternative model to analyse the gender difference in endurance performance because the gender difference can be analyzed in the same subjects collectively and also for the three disciplines separately (Knechtle, Knechtle, \& 
Lepers, 2011; Lepers, 2008; Lepers, \& Maffiuletti, 2011; Lepers, \& Stapley, 2011; Sultana, Brisswalter, Lepers, Hausswirth, \& Bernard, 2008).

Previous studies on the gender difference in triathlon performance have mainly focused on events lasting more than 8 hours, such as Ironman distance triathlons consisting in $3.8 \mathrm{~km}$ swimming, $180 \mathrm{~km}$ cycling and $42.2 \mathrm{~km}$ running (Lepers, 2008; Lepers, \& Maffiuletti, 2011) and ultra-triathlons (Knechtle, Knechtle, \& Lepers, 2011) or off-road triathlons (1.5 km swimming, $30 \mathrm{~km}$ mountain biking and $10 \mathrm{~km}$ trail running) (Lepers, \& Stapley, 2011). Surprisingly, little is known on the gender difference on more conventional short (Olympic) distance triathlon performance consisting in $1.5 \mathrm{~km}$ swimming, $40 \mathrm{~km}$ cycling and $10 \mathrm{~km}$ running.

The analysis of the Hawaii Ironman triathlon performances from 1988 to 2007 showed that the mean gender differences in elite (top ten overall) triathletes for swimming, cycling, running and total race time were $9.8 \%, 12.7 \%, 13.3 \%$ and $12.6 \%$, respectively (Lepers, 2008). The changes in the gender difference across the years differed between the three disciplines. Indeed, the gender difference in the swimming performance tended to remain stable across the 1998-2007 period, while it slightly increased in cycling and decreased in running (Lepers, 2008). The gender difference in the Hawaii Ironman triathlon performance seems to be greater in non-elite (age groups) triathletes compared to elite triathletes and is smaller for swimming compared to cycling and running. Indeed, Lepers and Maffiuletti (2011) reported gender differences of $12.1 \%$ for swimming, $15.4 \%$ for cycling, $18.2 \%$ for running and $15.8 \%$ for overall performance in age group Ironman triathletes. Moreover, the analysis of age and gender interactions showed that the gender difference in Ironman total performance time was stable until the age of 55 years and then significantly increased. 
Knowing that age and gender interactions in endurance performance could be influenced by the duration of the physical task performed (Tanaka, \& Seals, 1997), the combined interaction of age and gender on triathlon performance needs to be investigated for Olympic distance triathlon. Sultana et al. (2008) showed that for Olympic distance, the age-related decline in total performance appeared earlier in females (40 years) compared to males (45 years), but the gender differences in the age-related decline for the three disciplines have not been investigated by these authors.

In this context, the first aim of the present study was to examine the changes in male and female participation across years from 2000 to 2010 at the Olympic distance 'Zürich Triathlon'. According to the existing literature, we hypothesized an increase in the participation of female triathletes across the years. The second aim of this study was to examine the changes in the gender difference in Olympic distance triathlon performance for elite triathletes during the 2000-2010 period. Finally, the third aim was to compare the gender difference as a function of age among swimming, cycling, and running and overall race time during the same period. We hypothesized that the gender difference would differ between the locomotion modes and that the gender difference in total performance time would increase with advancing age. 


\section{Material and Methods}

Since 2000, the Olympic distance 'Zürich Triathlon' covering the distances of $1.5 \mathrm{~km}$ swimming, $40 \mathrm{~km}$ cycling and $10 \mathrm{~km}$ running has been held each year during months June, July or August. The swim course consists of one 1.5-km lap in the lake of Zurich with an average water temperature of $\sim 21^{\circ}$ Celsius. The cycle course consists of three flat laps equalling a total of $40 \mathrm{~km}$. Drafting is not allowed, a minimum distance of 7 meters must be held. The subsequent running course consists of two flat laps following the main road next to the lake equalling a $10 \mathrm{~km}$ run route. All race results from the 'Zürich Triathlon' from 2000 to 2010 were collected. The data set from this study was obtained from the race website (www.zueritriathlon.ch). This study was approved by the Institutional Review Board of St. Gallen, Switzerland, with waiver of the requirement for informed consent given that the study involved the analysis of publicly available data.

First, the age at the time of competition and swimming, cycling, running and total time performances of the elite i.e. top five male and female finishers overall at the 'Zürich Triathlon' were analysed from 2000 to 2010 (i.e. males, $n=55$; females, $n=55$ ). We chose to focus on the top five overall because it corresponded to the number of places with prize money. Second, swimming, cycling, running and total time performances of the top five of different age groups for males and females were analysed for the same period 2000-2010 (i.e. males, $n=450$; females, $n=360$ ). The age groups distinguish the categories for each period of years as follows: 18-24 years, 25-29 years, 30-34 years, 35-39 years, 40-44 years, 45-49 years, 50-54 years, 55-59 years, and 60-64 years. Age groups with less than five finishers in one year were not considered. For example, from 2000 to 2004, less than five females 
finished the race in the age group 50-54 years, so for the female age group 50-54 years, data were only considered from 2005 to 2010 .

The data of ages, swimming, cycling, running and total performance times were averaged across the first five male and female finishers for each year from 2000 to 2010. Swimming, cycling, running and total performance times were converted to minutes. The magnitude of the gender difference was examined by calculating the percent difference for the swimming, cycling, running and total times of the top five males versus females. One-way ANOVA showed that the mean time performance of the top five males and females of each age group for each individual discipline was not statistically different between years from 2000 to 2010, so we pooled 11 years of data. The percentage difference between the male and female in swimming, cycling, running and total event performance times for each of the top five places and for each year in each age group was determined.

Data are reported as means \pm standard deviation $(s)$ in the text. Linear regressions were used for estimating the changes of the selected variables per year. Pearson's correlation coefficients were used to assess the association between various variables (Statsoft, Version 6.1, Statistica, Tulsa, OK, USA). One-way ANOVA was used to compare top five gender differences in time across the three disciplines. To determine if the age of the top five male and the female finishers differed across the years, a separate ANOVA with repeated measures on year with gender as between-subject factor was performed. One-way ANOVA was used to compare gender difference in total time across ages. Two-way ANOVAs (age group $\times$ mode of locomotion) with repeated measures on locomotion mode were used to compare gender difference in time between swimming, cycling, and running across ages. Fisher's post hoc analyses were used to test differences within the ANOVAs when appropriate. A significance level of $P<0.05$ was used to identify statistical significance. 


\section{Results}

\section{Participation trends}

From 2000 to 2010, there were 7,939 total finishers (1,666 females and 6,273 males). Females accounted for $26.4 \pm 5.8 \%$ of the field since 2000 . The number of finishers each year over the history of the event is shown in Figure 1 Panel A. The age distribution of the finishers from 2000 to 2010 is displayed in Figure 1 Panel B. The 5-year age bracket with the largest participation has been from 35 to 39 years for males and from 30 to 34 years for females. Table 1 shows the linear regression of the participation trends per age group and year for both females and males from 2000 to 2010 . In males, no change in the number of participants was found whereas in females, the participation increased significantly for the age groups 40-44 years, 45-49 years and 50-54 years, respectively.

\section{Gender differences in performance for elite triathletes}

From 2000 to 2010 , there was a gender difference $\left(\mathrm{F}_{1,53}=4.72 ; P=0.032\right)$ in the age for the top five males and females. During this period, the mean age of the top five males $(26.8 \pm 5.7$ years) was significantly lower $(P<0.05)$ compared to the top five females $(28.4 \pm 5.8$ years $)$. Figure 2 shows the historical performance trends of the top five males and females between 2000 and 2010. Over this period, the mean swimming, cycling, running and overall race times were $20.9 \pm 1.8 \mathrm{~min}, 60.6 \pm 2.7 \mathrm{~min}, 34.0 \pm 1.2 \mathrm{~min}$, and $115.9 \pm 3.4 \mathrm{~min}$ for males, and $24.0 \pm 2.1$ min, 68.7 $\pm 3.8 \mathrm{~min}, 39.8 \pm 1.4 \mathrm{~min}$, and $133.1 \pm 3.7 \mathrm{~min}$ for females, respectively. Regression lines are presented from 2000 to 2010 for the three disciplines and overall race times for both genders. The slopes of the regression lines demonstrate that swimming and running performances did not really change since 2000 for both males and females. In contrast, the cycling time decreased significantly $(P<0.05)$ by $\sim 0.8$ min per annum for females and 
decreased by $\sim 0.5$ min per annum $(P=0.053)$ for males. During the $2000-2010$ period, females improved their overall race time by $\sim 0.8$ min per annum $(P<0.05)$, but overall race time for males remained stable. Figure 3 shows the change in the gender difference in time for swimming, cycling, running and overall race time for the top five finishers between 2000 and 2010. During this period, the gender differences in time showed no change for the three disciplines and for overall race time. The mean gender difference in time for swimming, cycling, running and overall race time were $15.2 \pm 4.6 \%, 13.4 \pm 2.3 \%, 17.1 \pm 2.5 \%$, and $14.8 \pm 1.8 \%$, respectively. ANOVA showed that the gender difference in cycling time was significantly $\left(\mathrm{F}_{2,163}=6.11 ; P=0.002\right)$ lower than for swimming and running.

\section{Age and gender interaction}

Table 2 represents the gender difference for males and females for both the split disciplines and overall race time for the different age groups. The gender difference in overall race time for the age groups 45-49 years and 50-54 years was significantly greater compared to the age groups 25-29 years and 30-34 years. The gender difference in overall race time for the age groups 35-39 years and 40-44 years was significantly greater compared to the age group 3034 years. For swimming, the gender difference in time for the age group 50-54 years was significantly greater compared to the age groups 18-24 years, 25-29 years and 30-34 years. The gender difference in time for the age groups 40-44 years and 45-49 years was significantly greater compared to the age group 30-34 years. No significant difference in the gender difference between the age groups was observed for cycling and running. Independently of the age, the gender differences in time differed between the locomotion modes. The gender difference in cycling time was significantly $\left(\mathrm{F}_{2,126}=11.04 ; P<0.001\right)$ lower than for swimming and running (Figure 4). 


\section{Discussion}

The first aim of the present study was to examine the changes in male and female participation across years from 2000 to 2010 at the Olympic distance 'Zürich Triathlon'. The results showed a significant increase in female participation but only for the age group triathletes between 40 and 54 years old and not for younger triathletes. The participation of male triathletes did not change during the studied period. It should be pointed out that the overall participation across the years remained quite stable during this period under the limit set by the organizer at 1,000 starters. An increased participation of female master athletes where a master athlete is defined as an athlete older than 35 years (Reaburn, \& Dascombe, 2008) has already been shown for half-marathoners (Leyk, et al., 2007), marathoners (Jokl, et al., 2004; Leyk, et al, 2007; Lepers, \& Cattagni, 2012) and ultra-marathoners (Hoffman, 2010; Hoffman, \& Wegelin, 2009). It is possible that females are gaining more competitive opportunities as they age, or that they are seeking out competition later in life, after childbirth, or later in their career (Ransdell, Vener, \& Huberty, 2009). Another aspect linked to the increase in participation of female triathletes is motivation. If winning prize money can be an additional motivation for elite triathletes, having fun and staying in good health may be the main motivations for recreational female triathletes. Additional studies would be useful to determine the main reasons for the increase in participation of female triathletes in competition and to verity if this finding is also observed for greater duration events such half and full Ironman distance triathlons.

The second aim of this study was to examine the changes in performance of male and female elite triathletes during the 2000-2010 period. The results showed that the female elite triathletes improved both their cycling time and their overall race time whereas the male elite 
triathletes showed no improvement in their performance. It seems that the top five overall female triathletes improved more in the cycling split across years thus leading to an improvement of their overall performance. Factors leading to an improved cycling performance in females compared with males could include an increased interest for training plans for female triathletes in relation with an increased acceptance and public interest of female sports participation over the last decades. The improvement in cycling times might also be due to changes and improvement in equipment (Bentley, Millet, Vleck, \& McNaughton, 2002), though this leads to improved performance in both males and females. The present study used linear regression to model times over increasing years, however, this model presents some limitations since; as the years pass the times would theoretically become negative. The analysis of performance speeds rather than times and asymptotic sigmoid models, as recommended by Nevill and Whyte (2005), could overcome these limitations and may well be an avenue for further research.

For the top five elite triathletes, the gender difference in the three split disciplines and overall race time did not significantly change between 2000 and 2010. The gender difference for swimming, cycling, running and overall race time was $15.2 \%, 13.4 \%, 17.1 \%$ and $14.8 \%$, respectively. Lepers (2008) investigated the gender difference for top ten triathletes competing in the 'Ironman Hawaii' from 1981 to 2007. The gender differences for swimming, cycling, running and overall race time were $9.8 \%, 12.7 \%, 13.3 \%$ and $12.6 \%$, respectively. Therefore, the gender differences were greater at the Olympic distance 'Zürich Triathlon' compared to the Hawaii Ironman triathlon for both the split disciplines and for overall race time. Obviously, female triathletes seem to reduce the gap in both split disciplines and overall performance with increasing length of a triathlon race. However, when we compare the gender difference of the Ironman distance with the World best performance times for longer triathlon distances such as a Double and Triple Iron ultra-triathlon, the gender difference 
increased with increasing length of an ultra-triathlon (Knechtle, et al., 2011). In elite Ironman triathletes, the gender difference tended to remain stable in swimming $(+0.1 \%$ per decade $)$ while it slightly increased in cycling $(+0.8 \%$ per decade) and decreased in running $(-2.8 \%$ per decade) across the years (Lepers, 2008). The absence of a change in the gender difference across the years in the present study might be due to the fact that we investigated elite athletes over a shorter distance within the time frame of only one decade, whereas Lepers (2008) investigated elite athletes over a longer 25-years time frame.

The third aim of the study was to examine the age and gender interactions in performance. We hypothesised that the gender difference would differ between the locomotion modes and that the gender difference would increase with advancing age. When considering all age groups, the gender difference was not different between swimming (18.5\%) and running (18.5\%), but the gender difference was significantly lower for cycling (15.5\%) compared to swimming and running. In contrast, it has been shown for the Hawaii Ironman triathlon that the gender difference in swimming $(12.1 \%)$ was significantly lower compared to cycling (15.4\%) and running (18.5\%), and that the gender difference in cycling was significantly lower compared to running (Lepers, \& Maffiuletti 2011). At the Olympic distance 'Zürich Triathlon', the gender difference was found to be the smallest for cycling (15.5\%) rather than for swimming as it was found at the Hawaii Ironman triathlon. The body composition of females compared to that of males may bring certain advantages for females in swimming endurance performance, resulting in a smaller gender difference in swimming endurance performance compared with cycling or running. For example, females held most of the world records in long-distance swimming in 1994 (Carbon, 1994). The physiology of this supremacy is not well understood but may relate to an improved fat metabolism, an improved local muscle endurance at low workloads, an improved tolerance of temperature extremes, and a greater buoyancy in water (Carbon, 1994). The greater body fat of females improves the 
buoyancy in the water and provides a better isolation against cold water, resulting in an advantage in swimming performance for females particularly in open water long-distance swimming with water temperatures of $10^{\circ}$ Celsius or less (Keatinge, Khartchenko, Lando, \& Lioutov, 2001; Knechtle, Christinger, Kohler, Knechtle, \& Rosemann, 2009). However, in the 1,500-m swim distance of an Olympic distance triathlon with the water temperature in the lake Zurich being around $20^{\circ}$ Celsius, the greater body fat may be not a real advantage for females.

The gender difference in running at the Olympic distance 'Zürich Triathlon' was $~ 18.5 \%$, which is equivalent to the gender difference in running at the long distance Hawaii Ironman triathlon with $18.5 \%$ (Lepers, \& Maffiuletti, 2011). This finding is inconsistent with the thesis that the gender difference may disappear with increasing running distance (Beneke, Leithäuser, \& Doppelmayer (2005). For example, in the 'Badwater' ultra-marathon, a $216 \mathrm{~km}$ race at temperatures of up to $55^{\circ}$ Celsius (www.badwater.com), female athletes became faster than the male competitors (Beneke, et al., 2005). Males dominated the race during the 1980s and 1990s, but consistent male superiority is already a matter of history. In 2002 and 2003, a female ultra-runner outpaced the fastest male by about 4.5 and 0.5 hours, respectively. Furthermore, since 2002, up to three females have been in the first five finishers, even though there were more male than female participants (Beneke, et al., 2005).

Support for this assumption of a closing gender gap over long-distance races comes from findings such as gender differences in the ability to run aerobically at a higher percent of maximum oxygen uptake $\left(\mathrm{VO}_{2} \mathrm{max}\right)$, the use of ingested and stored glycogen, lipid metabolism and possibly the acute response of growth hormones in the balance between gene transcription, translation, protein breakdown, resistance to oxidative stress and also pain perception (Beneke, et al., 2005). For swimming, Tanaka and Seals (1997) showed that the 
gender difference in performance became smaller with increasing distances from 50m to 1,500m. Tanaka and Seals (1997) analysed swimming performances and showed that in both males and females the endurance swimming performance $(1,500 \mathrm{~m})$ declined linearly from peak levels at age 35-40 years until 70 years of age, and declined exponentially thereafter. The rate and the magnitude of the declines in both short- and long-duration swimming performance with age were significantly greater in females than in males. The percent gender difference in swimming performance throughout the age range studied became progressively smaller with increasing distance from $50 \mathrm{~m}$ to $1,500 \mathrm{~m}$ (Tanaka, \& Seals, 1997). In contrast to studies describing the gender difference decreases with longer running distances, Knechtle, Knechtle, and Lepers (2011 stated that the gender difference in ultra-triathlons increased with increasing ultra-distance, in a Double and a Triple Iron ultra-triathlon $\sim 20 \%$, and in a Deca Iron ultra-triathlon $\sim 30 \%$, respectively.

The results of our analysis showed that the gender difference in overall performance significantly increased after the age of 35 years. The gender difference in swimming increased significantly after the age of 40 years but remained similar for cycling and running with advancing age. These findings differ from those of Lepers and Maffiuletti (2011) reporting for Ironman triathletes a stability of the gender difference for overall race time until the age of 55 years and an increase thereafter. Possible explanations could be that elderly female triathletes are more competitive at the Hawaii Ironman triathlon since it is a World Championship event, and also that only some of the fastest and best trained female triathletes qualify for the Hawaii ironman triathlon. For recreational triathletes, an Olympic distance triathlon can be finished within 2-3 hours whereas it takes 12 hours and more to finish an Ironman triathlon (Lepers, 2008; Lepers, \& Maffiuletti, 2011). 
The age-related decline in performance is well known and declines in athletic performance are inevitable with aging. The decline is mostly due to changes in physiological factors and modulated by lifestyle, training and nutritional factors (Leyk, et al., 2009; Tanaka, \& Seals, 2003; Tanaka, \& Seals, 2008; Trappe, 2007). Tanaka and Seals (2008) described the changes in peak endurance exercise performance with advancing age as well as physiological factors responsible for those changes. In agreement with our findings in this study of triathlon performance, they found peak endurance performance to be maintained until $\sim 35$ years of age, followed by a moderate decrease until 50-60 years of age, with progressively steeper declines thereafter. Among the three main physiological determinants of endurance exercise performance $\left(\mathrm{VO}_{2} \max\right.$, lactate threshold and exercise economy), a progressive reduction in $\mathrm{VO}_{2} \mathrm{max}$ appears to be the primary mechanism associated with declines in endurance performance with age (Tanaka, \& Seals, 2008). Reductions in the lactate threshold were also found to contribute, whereas submaximal exercise economy was preserved with ageing in endurance athletes (Tanaka, \& Seals, 2008). The age-associated decreases in $\mathrm{VO}_{2}$ max in endurance exercise-trained adults are mediated by reductions in maximal cardiac output and maximal arterio-venous $\mathrm{O}_{2}$ difference, with reductions in both maximal stroke volume and heart rate contributing to the former (Tanaka, \& Seals, 2008).

Aging as a natural process can be considerably speeded up or slowed by lifestyle (Leyk, et al., 2009; Trappe, 2007). The training status of master athletes is an important modulator of performance loss with advancing age. The changes in physiological function and running performance with age are closely related to the level of distance run training (Trappe, 2007). Current research supports the concept that continued running late into life attenuates a decline in physiological function with age and is beneficial for overall health (Trappe, 2007). Leyk et al. (2009) also investigated lifestyle factors influencing the age-related performance decline. In contrast to previous studies, they found no age-related losses in endurance performance 
starting after the age of 30 years. Instead, they stated that significant age-related declines in marathon performance did not occur before the age of $\sim 55$ years.

It is often mentioned that sprinting performance is more affected by increasing age than endurance performance. However, past analyses of master athletic world record data have yielded opposite observations. Rittweger, Di Pampero, Maffulli and Narici (2009) showed that when assessing the metabolic power required achieving running world records, sprint and endurance events showed a relatively uniform decline with age across the different events. Other studies described the overall reduction in peak exercise performance with age tending to be greater in endurance than in sprint events (Tanaka, \& Seals, 1997; Tanaka, \& Seals, 2003). It is not entirely known why or how the duration of the athletic event influences the age-associated decline in peak performance. One possibility is that endurance and sprint events rely on different energy-producing pathways to sustain muscle activity. Wright and Perricelli (2008) reported no difference in the decline of sprint and endurance events in males, whereas the decline in the sprint was greater than in endurance for females, especially after the age of 75 years. An interesting implication for futures studies would be to compare sprint and endurance performance decline with advancing age of the same individual.

\section{Conclusions}

From 2000 to 2010, female triathletes aged from 40 to 54 years increased their participation at the Olympic distance 'Zürich Triathlon' while the participation of younger females and males remained stable. During this period, female and male overall race performance time did not significantly change except for the males in the age group 50-54 years and for the females in the age group 45-49 years that improved their total race time. For elite triathletes, the gender differences in time did not significantly change for the three disciplines and for overall race time across the studied period. However, the gender difference in Olympic distance triathlon 
performance increased after the age of 35 years which appeared earlier compared to previous data on long distance triathlon (Lepers \& Maffiuletti, 2011). Future studies should compare gender difference in performance across the age for different endurance events to confirm a possible effect of exercise duration on gender difference with advancing age. 


\section{References}

Bam, J., Noakes, T. D., Juritz, J., \& Dennis, S. C. (1997). Could women soon outrun men in ultramarathon races? Medicine \& Science in Sports \& Exercise, 29, 244-247.

Beneke, R., Leithäuser, R. M., \& Doppelmayer, M. (2005). Women will do it in the long run. British Journal of Sports Medicine, 39, 410.

Bentley, D. J., Millet, G. P., Vleck, V. E., \& McNaughton, L. R. (2002). Specific aspects of contemporary triathlon: implications for physiological analysis and performance. Sports Medicine, 32, 345-359.

Carbon, R. (1994). ABC of Sports Medicine: Female Athletes. British Medical Journal, 309, 254-258.

Cheuvront, S. N., Carter, III R., DeRuisseau, K. C., \& Moffart, R. J. (2005). Running performance differences between men and women. An update. Sports Medicine, 35, 10171024.

Coast, J. R., Blevins, J. S., \& Wilson, B. A. (2004). Do gender differences in running performance disappear with distance? Canadian Journal of Applied Physiology, 29, 139-145.

Hoffman, M. D. (2010). Performance trends in 161-km ultramarathons. International Journal of Sports Medicine, 31, 31-37.

Hoffman, M. D., \& Wegelin, J. A. (2009). The Western States 100-Mile Endurance Run: participation and performance trends. Medicine and Science in Sports and Exercise, 41, 21912198.

Jokl, P., Sethi, P. M., \& Cooper, A. J. (2004). Master's performance in the New York City Marathon 1983-1999. British Journal of Sports Medicine, 38, 408-412.

Keatinge, W. R., Khartchenko, M., Lando, N., \& Lioutov, V. (2001). Hypothermia during sports swimming in water below 11 degrees C. British Journal of Sports Medicine, 35, 352353.

Knechtle, B., Christinger, N., Kohler, G., Knechtle, P., \& Rosemann, T. (2009). Swimming in ice cold water. Irish Journal of Medical Science, 178, 507-511.

Knechtle, B., Knechtle, P., \& Lepers, R. (2011). Participation and performance trends in ultratriathlons from 1985 to 2009. Scandinavian Journal of Medicine and Science in Sports, 21, e82-e90.

Lepers, R. (2008). Analysis of Hawaii ironman performances in elite triathletes from 1981 to 2007. Medicine and Science in Sports and Exercise, 40, 1828-1834.

Lepers, R., \& Cattagni, T. (2012). Do older athletes reach limits in their performance during marathon running? Age (Dordr), 34, 773-781. 
Lepers, R., \& Stapley, P. J. (2011). Age-related changes in conventional road versus off-road triathlon performance. European Journal of Applied Physiology, 111, 1687-1694.

Lepers, R., \& Maffiuletti, N. A. (2011). Age and gender interactions in ultraendurance performance: insight from the triathlon. Medicine and Science in Sports and Exercise, 43, 134-139.

Lepers, R., Sultana, F., Bernard, T., Hausswirth, C., \& Brisswalter, J. (2010). Age-related changes in triathlon performances. International Journal of Sports Medicine, 31, 251-256.

Leyk, D., Erley, O., Ridder, D., Leurs, M., Rüther, T., Wunderlich, M.. Sievert, A., Baum, K., \& Essfeld, D. (2007). Age-related changes in marathon and half-marathon performances. International Journal of Sports Medicine, 28, 513-517.

Leyk, D., Erley, O., Gorges, W., Ridder, D., Rüther, T., Wunderlich, M., Sievert, A., Essfeld, D., Piekarski, C., \& Erren, T. (2009). Performance, training and lifestyle parameters of marathon runners aged 20-80 years: results of the PACE-study. International Journal of Sports Medicine, 30, 360-365.

Nevill, A.M., \& Whyte, G. (2005) Are there limits to running world-records? Medicine and Science in Sports and Exercise, 37, 1785-1788.

Ransdell, L. B., Vener, J., \& Huberty, J. (2009). Master athletes : An analysis of running, swimming and cycling performance by age and gender. Journal of Exercise Science and Fitness, 7, 61-73.

Reaburn, P., \& Dascombe, B. (2008). Endurance performance in master athletes. European Review of Aging and Physical Activity, 5, 31-42.

Rittweger, J., Di Pampero, P. E., Maffulli, N., \& Narici, M. V. (2009). Sprint and endurance power and ageing: an analysis of master athletic world records. Proceedings of the Royal Society, 276, 683-689.

Seiler, S., De Koning, J. J., \& Foster, C. (2007). The fall and rise of gender difference in elite anaerobic performance 1952-2006. Medicine and Science in Sports and Exercise, 39, 534540.

Sparling, P. B., O’Donnell, E. M., \& Snow, T. K. (1998). The gender difference in distance running has plateaued: analysis of world rankings from 1980 to 1996. Medicine and Science in Sports and Exercise, 30, 1725-1729.

Sultana, F., Brisswalter, J., Lepers, R., Hausswirth, C., \& Bernard, T. (2008). Effects of age and gender on Olympic triathlon performances. Science \& Sports, 23, 130-135.

Tanaka, H., \& Seals, D. R. (1997). Age and gender interactions in physiological functional capacity: insight from swimming performance. Journal of Applied Physiology, 82, 846-851.

Tanaka, H, \& Seals, D. R. (2003). Invited Review: Dynamic exercise performance in masters athletes: insight into effects of primary human aging on physiological functional capacity. Journal of Applied Physiology, 95, 2152-2162. 
Tanaka, H, \& Seals, D. R. (2008). Endurance exercise performance in masters athletes: ageassociated changes and underlying physiological mechanisms. Journal of Physiology, 586, 55-63.

Trappe, S. (2007). Marathon runners: how do they age? Sports Medicine, 37, 302-305.

Whipp, B. J., \& Ward, S. A. (1992). Will women soon outrun men? Nature, 355, 25.

Wright, V. J., \& Perricelli, B. C. (2008). Age related rates of decline in performance among elite senior athletes. American Journal of Sports Medicine, 36, 443-450. 


\begin{tabular}{|c|c|c|c|c|c|c|c|c|c|c|c|c|c|c|c|c|c|c|}
\hline & \multicolumn{18}{|c|}{ Age groups (years) } \\
\hline & \multicolumn{2}{|c|}{$18-24$} & \multicolumn{2}{|c|}{$25-29$} & \multicolumn{2}{|c|}{$30-34$} & \multicolumn{2}{|c|}{$35-39$} & \multicolumn{2}{|c|}{$40-44$} & \multicolumn{2}{|c|}{$45-49$} & \multicolumn{2}{|c|}{$50-54$} & \multicolumn{2}{|c|}{$55-59$} & \multicolumn{2}{|c|}{$60-64$} \\
\hline & $r^{2}$ & $P$ & $r^{2}$ & $P$ & $r^{2}$ & $P$ & $r^{2}$ & $P$ & $r^{2}$ & $P$ & $r^{2}$ & $P$ & $r^{2}$ & $P$ & $r^{2}$ & $P$ & $r^{2}$ & $P$ \\
\hline Males & 0.005 & 0.829 & 0.186 & 0.185 & 0.041 & 0.549 & 0.015 & 0.716 & 0.131 & 0.275 & 0.286 & 0.090 & 0.354 & 0.053 & 0.014 & 0.728 & 0.227 & 0.138 \\
\hline Females & 0.088 & 0.377 & 0.089 & 0.374 & 0.160 & 0.223 & 0.096 & 0.353 & 0.646 & 0.003 & 0.734 & 0.001 & 0.631 & 0.004 & 0.304 & 0.079 & 0.150 & 0.239 \\
\hline
\end{tabular}

Table 1: Linear regressions corresponding to the changes in the number of male and female finishers of the different age-groups across the studied time period (2000-2010) at the Olympic distance 'Zürich Triathlon' 


\section{Age groups (years)}

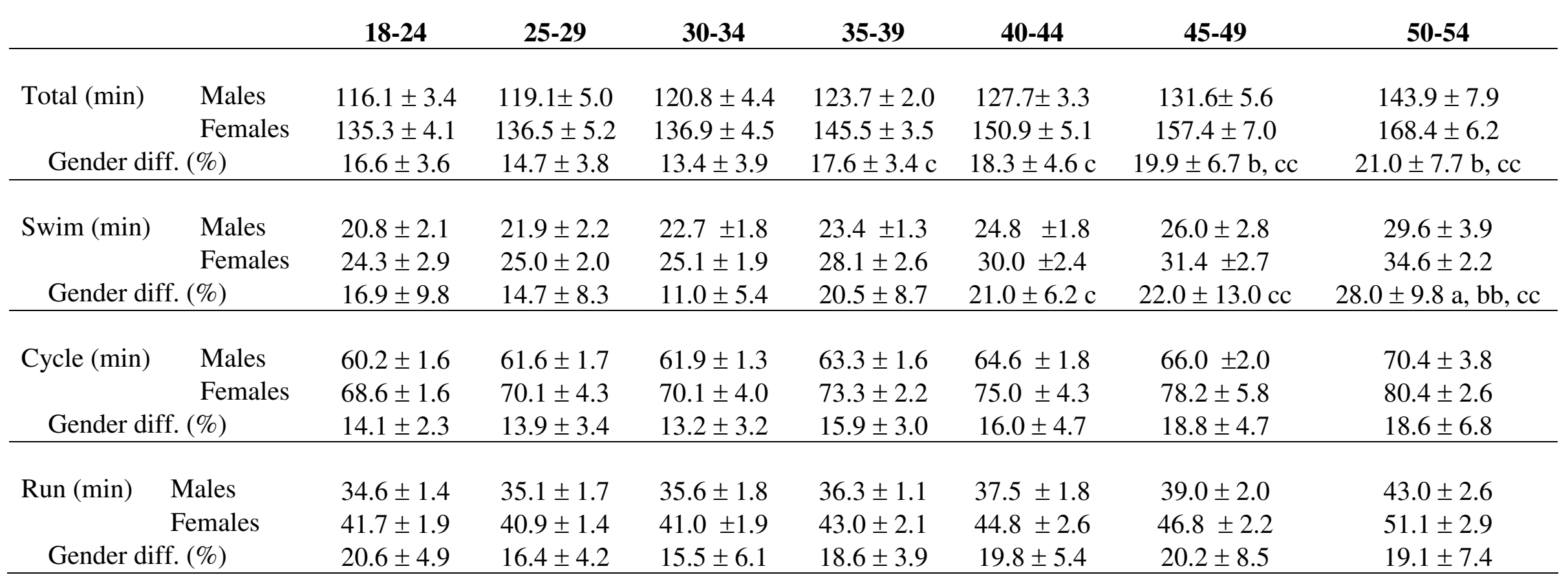

Table 2: Total, swimming, cycling, and running performance times (min) and corresponding gender differences for the top five males and females in each age group at the Olympic distance 'Zürich Triathlon' ( $1.5 \mathrm{~km}$ swim, $40 \mathrm{~km}$ cycle and $10 \mathrm{~km}$ run). Data were pooled from 2000 to 2010 years. Data for females in the age group 50 to 54 years were only available from 2005 to 2010 .Values are presented as mean $\pm s$.

a: significantly different from $18-24$ years $; P<0.05$; aa: significantly different from 18-24 years; $P<0.01$

b: significantly different from 25-29 years ; $P<0.05$; bb: significantly different from 25-29 years; $P<0.01$

c: significantly different from 30-34 years ; $P<0.05$; cc: significantly different from 30-34 years; $P<0.01$ 


\section{Figure legends}

Figure 1 Number of finishers at the Olympic distance 'Zürich Triathlon' of each gender from 2000 to 2010 (panel A). Age distribution $($ mean $\pm s$ ) of female and male finishers from 2000 to 2010 (panel B).

Figure 2 Swimming, cycling, running and total performance times (mean $\pm s$ ) at the Olympic distance 'Zürich Triathlon' for the top five male and female finishers from 2000 to 2010 . The years analysed are pooled and the mean values of males and females are shown on the right side of the panel (Total).

Figure 3 Gender differences between the top five male and female finishers for swimming, cycling, running, and total times from 2000 to 2010 at the Olympic distance 'Zürich Triathlon' (mean $\pm \mathrm{SE}$ ). The white circles on the right side represent the mean gender difference of the all years (Total).

Figure 4 Averaged gender difference in time performance for swimming, cycling, running, and total event across all age groups (20-24 years to 50-54 years) at the Olympic distance 'Zürich Triathlon'; data were pooled from 2000 to 2010. Values are means \pm SE. ***: Significantly different from cycle, $P<0.001$ 

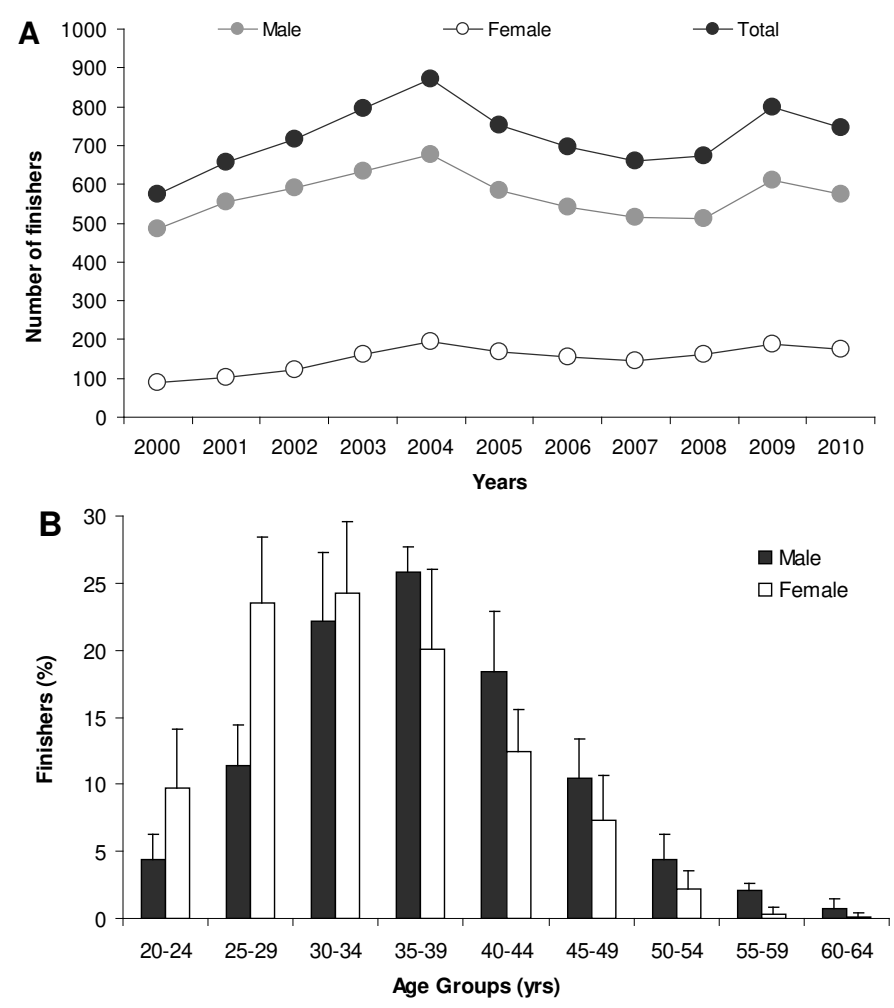

Figure 1 

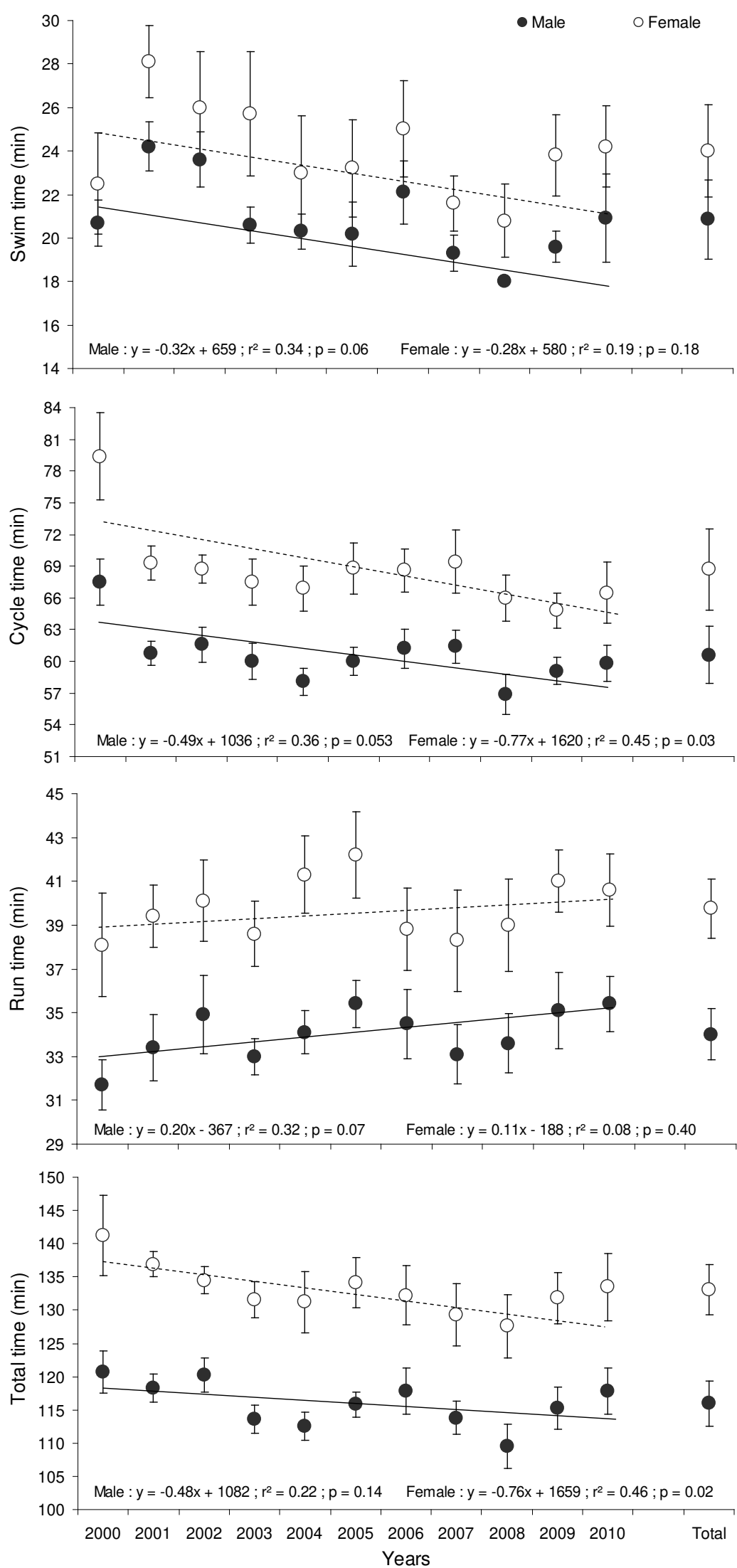

Figure 2 

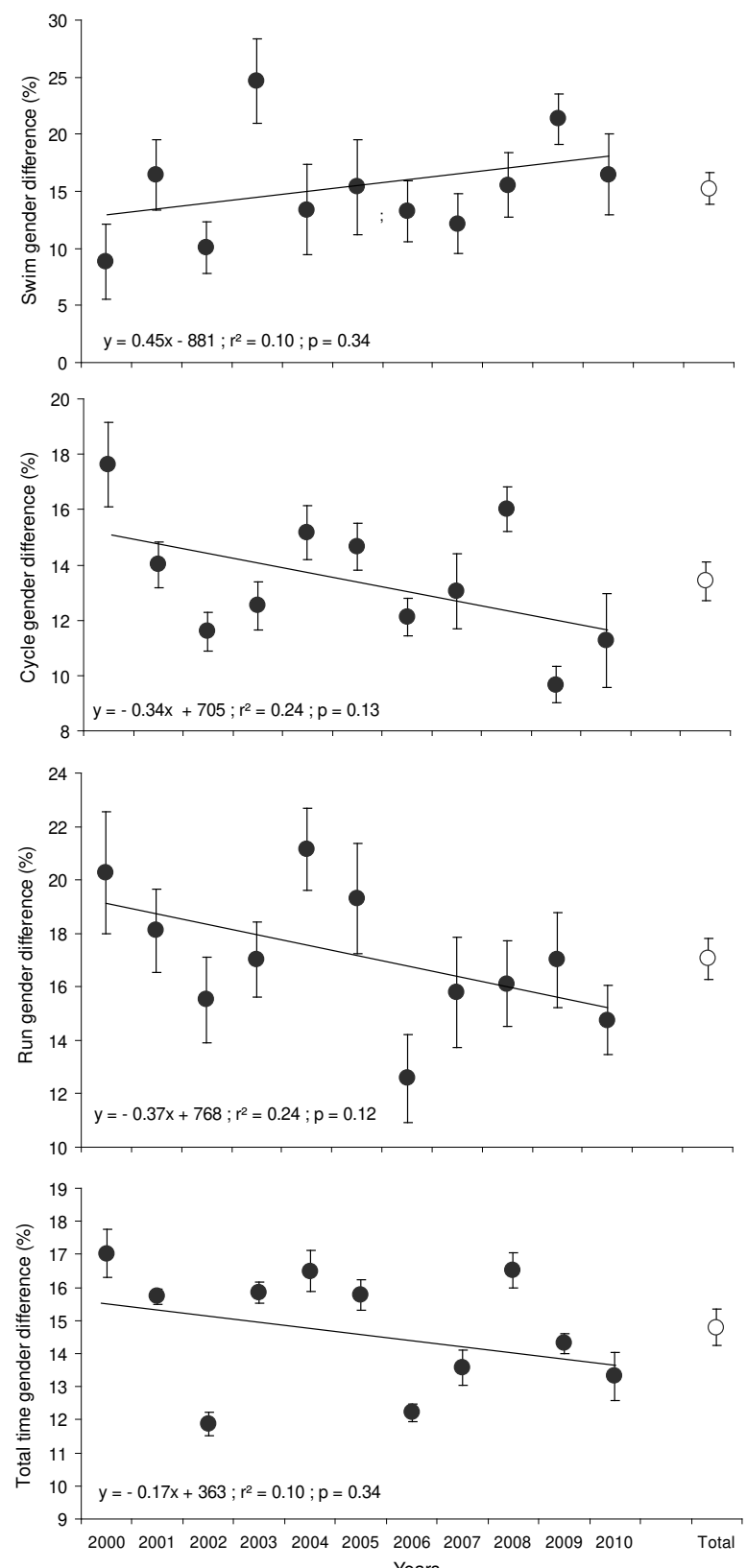

Figure 3 


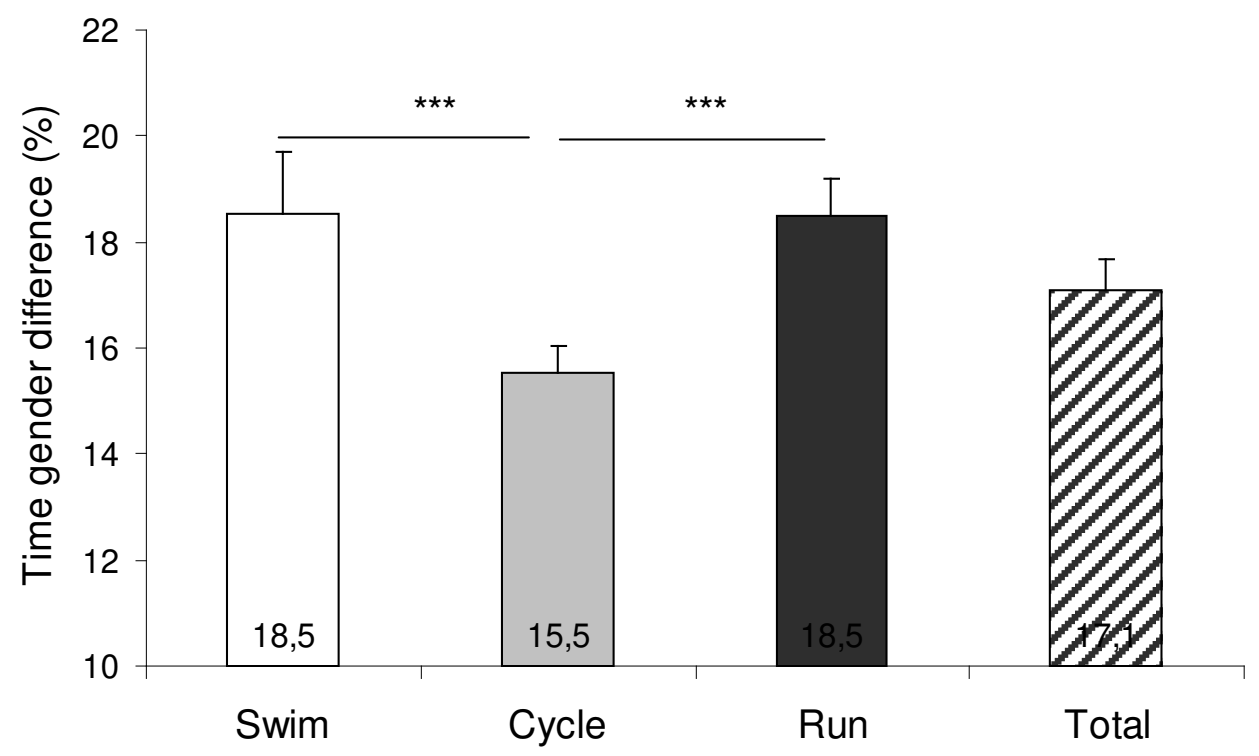

Figure 4 